\title{
Determination and mapping of diameter and helicity for single-walled carbon nanotubes using nanobeam electron diffraction
}

\author{
Zejian Liu, ${ }^{1}$ Qi Zhang, ${ }^{1}$ and Lu-Chang Qin ${ }^{1,2, *}$ \\ ${ }^{1}$ Department of Physics and Astronomy, University of North Carolina at Chapel Hill, Chapel Hill, North Carolina 27599-3255, USA \\ ${ }^{2}$ Curriculum in Applied and Materials Sciences, University of North Carolina at Chapel Hill, Chapel Hill, North Carolina 27599-3255, USA
}

(Received 16 December 2004; revised manuscript received 9 March 2005; published 22 June 2005)

\begin{abstract}
The atomic structures of 124 single-walled carbon nanotubes, described by their diameter and helicity or equivalently by the two chiral indices $(u, v)$ that define the perimeter of each nanotube, have been determined unambiguously by nanobeam electron diffraction. A mapping of 70 possible nanotubes in the range of 1.20 $1.65 \mathrm{~nm}$ in diameter with all possible helicities has been constructed experimentally for a carbon nanotube sample produced by arc discharge. Among the total 124 nanotubes characterized experimentally, 58 nanotubes of different structure have been identified. By examining the histogram of occurring helicities, we find that, while certain nanotubes were observed slightly more often than others, the overall feature showed a rather uniform distribution in occurrence. Basing on a nucleation-and-growth model, we suggest that the uniform distribution of helicity be originated from the weak dependence on helicity of the formation energy of carbon nanotubes, while the growth prefers slightly the structure with helicity $15^{\circ}-30^{\circ}$ for which the addition of carbon dimers would facilitate the growth of carbon nanotubes.
\end{abstract}

DOI: 10.1103/PhysRevB.71.245413

PACS number(s): 61.46.+w, 61.14.Lj, 81.07.De

\section{INTRODUCTION}

Carbon nanotubes are a promising material for various nanotechnological applications such as field-induced electron emitters, sensors, and components of molecular electronic circuits. The properties of a carbon nanotube depend on its atomic structure. A typical example is the electronic structure of a carbon nanotube-depending on its diameter and helicity, the carbon nanotube can be either metallic or semiconducting. ${ }^{1,2}$ In terms of the crystallographic indices $(u, v)$, which define the perimeter vector $\vec{C}_{h}$ on graphene by $\vec{C}_{h}=u \vec{a}_{1}+v \vec{a}_{2}\left(\vec{a}_{1}\right.$ and $\vec{a}_{2}$ are the crystallographic basis vectors of graphene with the amplitude of $a_{0}=0.2461 \mathrm{~nm}$ and an interangle of $60^{\circ}, u$ and $v$ are integers), if $u-v$ is divisible by 3 , the nanotube is metallic and otherwise it is semiconducting unless the diameter is extremely small where all nanotubes appear metallic. ${ }^{3}$ Of all possible carbon nanotube species, about two thirds are semiconducting and one third metallic.

A great deal of effort has been devoted to the determination of the $(u, v)$ indices and their distribution ever since the discovery of carbon nanotubes. Several analytical techniques, including both global and local probe techniques such as Raman spectroscopy, ${ }^{4,5}$ optical absorption and luminescence, ${ }^{6,7}$ and scanning tunneling microscopy, ${ }^{8,9}$ have been applied to map the $(u, v)$ indices. However, the effectiveness in assigning the $(u, v)$ indices unambiguously by these techniques has been limited by their resolution. On the other hand, electron diffraction, in particular when a nanobeam electron probe is used, i.e., nanobeam electron diffraction (NBED), can now provide accurate measurement of both the helicity and diameter of carbon nanotubes. ${ }^{10-17}$ Comparing with other techniques, the NBED technique offers advantages in not only more accurate deduction of helicity and diameter for individual nanotubes but also that the individual nanotube can be imaged at the same time. With the determination of helicity and diameter of each individual carbon nanotube, the unique structure $(u, v)$ can be assigned.

We have examined 124 individual single-walled carbon nanotubes (SWNTs) and obtained their diameter and helicity unambiguously using NBED from a sample produced by arc discharge. ${ }^{18}$ Carbon nanotubes in this sample usually contain a large amount of impurities including metal particles, graphitic particles, and amorphous carbon. In addition, SWNTs usually aggregate to form raftlike bundles or ropes due to the van der Waals interactions between the tubule walls. ${ }^{19}$

\section{THEORY}

Due to the finite size of carbon nanotubes in the radial direction, the intensity distribution of the electron diffraction pattern from a SWNT deviates noticeably from that of graphene. The electron diffraction intensities elongate perpendicular to the tubule axis and form a set of diffraction layer lines. Kinematical theory of electron diffraction can be used to interpret the electron diffraction patterns of carbon nanotubes because of the low atomic number of carbon and the thin layers of the tubule. ${ }^{20,21}$ The scattering amplitude for a SWNT can be expressed as

$$
\begin{aligned}
F(R, \Phi, l)= & \sum_{n, m} \exp [\operatorname{in}(\Phi+\pi / 2)] J_{n}(\pi d R) \\
& \times \sum_{j} f \exp \left[i\left(-n \phi_{j}+2 \pi l z_{j} / c\right)\right],
\end{aligned}
$$

where $d$ is the diameter of the nanotube defined as $d$ $=\sqrt{u^{2}+v^{2}+u v} a_{0} / \pi, f$ is the atomic scattering amplitude of carbon for electrons, and $c$ is the axial periodicity of the nanotube. The summation for $j$ is done over all the composing helices for the nanotube with the relative atomic shifts of $\left(d / 2, \phi_{j}, z_{j}\right)$ in the cylindrical coordinates and for $n$ and $m$ over all integers as allowed by the selection rule $l=n c / C$ 
$+m c / \Delta$ with $C$ being the pitch length of the helices and $\Delta$ being the axial distance between neighboring carbon atoms along a helix. For a SWNT of chiral indices $(u, v)$ with the perimeter length of $C_{h}$ and helicity of $\alpha, C=C_{h} \tan \left(60^{\circ}-\alpha\right)$ and $\Delta=a_{0} \sin \left(60^{\circ}-\alpha\right)$. With the axial periodicity derived as $c=\sqrt{3} C_{h} / M$ where $M$ is the maximum common divisor of $(2 u+v)$ and $(u+2 v)$, we can derive the selection rule for a SWNT $(u, v)$ as

$$
l=\left[n(u+2 v)+2 m\left(u^{2}+v^{2}+u v\right)\right] /(u M) .
$$

Considering the geometrical relationships between all the composing helices of a $\operatorname{SWNT}(u, v)$, the scattering amplitude can be expressed as ${ }^{22}$

$$
\begin{aligned}
F_{u v}(R, \Phi, l)= & \sum_{n, m} f \chi_{u v}(n, m) \gamma_{u v}(n, m) \\
& \times \exp \left[i n\left(\Phi+\frac{\pi}{2}\right)\right] J_{n}(\pi d R),
\end{aligned}
$$

where we have defined $\chi_{u v}(n, m)$ and $\gamma_{u v}(n, m)$ as

$$
\chi_{u v}(n, m)=1+\exp \{2 \pi i[n+(2 u+v) m] / 3 u\},
$$

and

$$
\begin{aligned}
\gamma_{u v}(n, m) & =\frac{1-\exp [-2 \pi i(n+m v)]}{1-\exp [-2 \pi i(n+m v) / u]} \\
& = \begin{cases}u, & (n+m v) / u=\text { integer } \\
0, & \text { otherwise }\end{cases}
\end{aligned}
$$

The diffraction intensity distribution is then dictated by

$$
I_{u v}(R, \Phi, l)=\left|F_{u v}(R, \Phi, l)\right|^{2} .
$$

From Eqs. (2)-(6), we can see that the intensity distribution on each layer line is modulated by Bessel functions of various orders, which account for the curvature of the cylindrical tubule and are dominated by a single Bessel function of the order determined by the selection rule. On the equatorial layer line where $l=0$, the selection rule in Eq. (2) dictates that the dominating order of Bessel function is $n=0$, i.e., the intensity distribution on the equatorial layer line is modulated by $\left|J_{0}(\pi d R)\right|^{2}$, from which we can determine accurately the diameter $d$ of a SWNT $(u, v)$.

As regards to the scattering intensities on the nonequatorial layer lines, it is of special interest to examine the layer lines corresponding to the principal reflections of $\{100\}^{*}$ type, which bear the strongest intensities in the electron diffraction patterns of carbon nanotubes. The three principal layer lines, due to the $\{100\}$-type reflections of the graphene structure (cf. Fig. 1), lying above the equatorial layer line are referred to as $L_{1}, L_{2}$, and $L_{3}$ in descending order and their values are $l_{1}=(2 u+v) / M, l_{2}=(u+2 v) / M$, and $l_{3}=(u-v) / M$, respectively. ${ }^{13,23}$ By reference to Eqs. (2) and (5), we can obtain that the orders of Bessel functions that dominate the intensity distributions on layer line $l_{1}, l_{2}$, and $l_{3}$ are $n=-v$, $n=u$, and $n=-(u+v)$, respectively. Therefore, the scattering intensities on the three principal layer lines $L_{1}, L_{2}$, and $L_{3}$ are

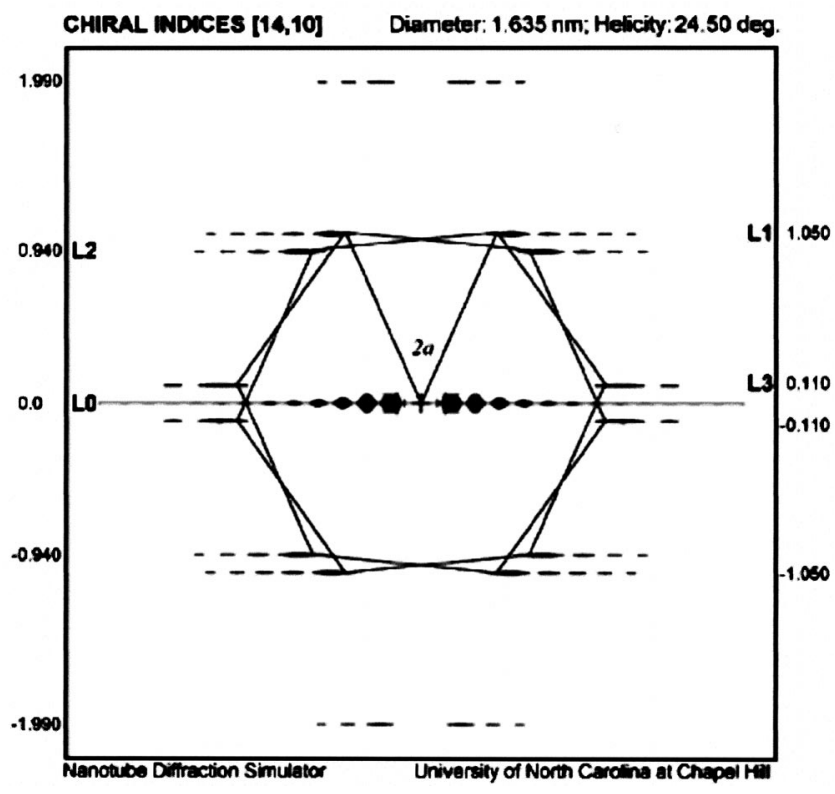

FIG. 1. Calculated electron diffraction pattern of carbon nanotube of chiral indices $(14,10)$. The two hexagons represent the primary $\{100\}$-type reflections from graphene which form principal layer lines labeled as $L_{1}, L_{2}$, and $L_{3}$ as indicated in the figure. The intensities are elongated in the direction perpendicular to the tubule axis and the center of each reflection also shifts outward compared with the corresponding graphene reflection.

$$
\begin{gathered}
I\left(R, \Phi, l_{1}\right) \propto\left|J_{v}(\pi d R)\right|^{2}, \\
I\left(R, \Phi, l_{2}\right) \propto\left|J_{u}(\pi d R)\right|^{2}, \\
I\left(R, \Phi, l_{3}\right) \propto\left|J_{u+v}(\pi d R)\right|^{2} .
\end{gathered}
$$

Figure 1 shows the calculated electron diffraction pattern of carbon nanotube of chiral indices $(14,10)$. The overlapped hexagons represent the primary graphene reflections and the angle $\alpha$ is the helicity of the nanotube.

On the other hand, it should be noted that Eqs. (7a)-(7c) also offer a precise and rapid method to determine the chiral indices of single-walled carbon nanotubes directly. Since the positions of the intensity peaks are unique to each order of the Bessel function, we can identify the order of the Bessel function that matches the diffraction intensities on these three principal layer lines. These chiral indices $(u, v)$ can therefore be readily assigned unambiguously once the corresponding order of the Bessel function is determined from the experimental electron diffraction pattern.

\section{EXPERIMENT}

High-resolution NBED data were acquired from a transmission electron microscope equipped with a field-emission gun (JEM-2010F). To avoid radiation damage to the SWNT structure, ${ }^{24}$ the microscope was operated at $80 \mathrm{kV}$ and a parallel electron illumination with $20 \mathrm{~nm}$ beam size was obtained by utilizing a $10 \mu \mathrm{m}$ condenser aperture and exciting the first condenser lens to maximum. The NBED patterns of 
(a)

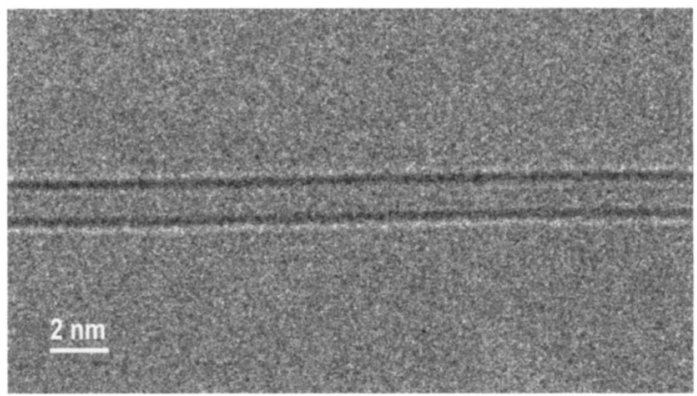

(b)
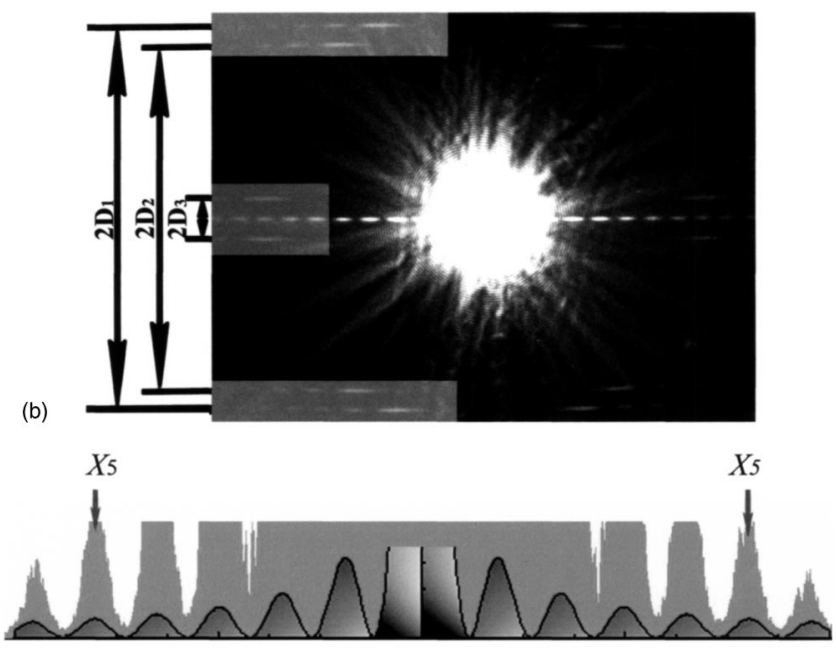

(c)

FIG. 2. Characterization of a chiral SWNT $(14,10)$. (a) HRTEM image of the SWNT. The nanotube diameter, $1.60 \pm 0.08 \mathrm{~nm}$, was measured directly from the two dark lines. (b) Corresponding NBED pattern. The helicity of this nanotube is measured $24.50^{\circ} \pm 0.08^{\circ}$ from the layer line spacings. (c) Digitized diffraction intensity profile of the equatorial layer line in (b). The distance $2 R$ between the two peaks $\left(X_{5}\right)$ indicated by arrows is used to measure the nanotube diameter. The overlapped intensity profile is the calculated electron intensities expressed by the module square of the zero-order Bessel function $J_{0}(\pi d R)$.

individual SWNTs were recorded on photographic films, which were later digitized using a high resolution film scanner.

Figure 2(a) shows a typical high-resolution transmission electron microscope (HRTEM) image of a SWNT, in which the dark lines correspond to the projection of the side walls of the nanotube and the chirality of the nanotube is usually not reflected. The corresponding NBED pattern, given in Fig. 2(b), consists of several layer lines due to the periodicity of the structure in the axial direction of the nanotube. The diameter of the nanotube, about $1.60 \mathrm{~nm}$, can be measured directly from the image using the graphitic spacing $(0.345$ nm) as a scale bar.

However, more accurate measurement of the diameter can be obtained from the electron diffraction intensity distribution on the equatorial layer line as discussed in the above section. The diffraction intensity distribution on the equatorial layer line is modulated mainly by the module square of the zero-order Bessel function $J_{0}(X)(X=\pi d R)$ as shown in the central layer line in Fig. 2(b). Figure 2(c) shows the corresponding intensity profile of the equatorial layer line.
Measurement of the ratio of the two peak positions (the fifth maximum of the module square of zero-order Bessel function on each side) on the equatorial layer line in reciprocal space (the distance between them is $2 R$, indicated by dark arrows) was used to deduce the diameter $d$ of the nanotube

$$
d=X_{5} / \pi R
$$

where $X_{5}$ is the value at which $\left|J_{0}(X)\right|^{2}$, the module square of the zero-order Bessel function, acquires its fifth maximum. The diameter $d$ of this carbon nanotube measured from the equatorial layer line in its electron diffraction pattern is 1.60 $\mathrm{nm}$. In our experiment, since the maximum error $\sigma_{R}$ in the measurement of the peak position $R$ was $1 \%$, the maximum error $\sigma_{d}$ of measuring the nanotube diameter $d$ is also $1 \%$ $\left(\sigma_{d} / d=\sigma_{R} / R\right)$. The overlapped intensity profile is the simulated intensity of the module square of the zero-order Bessel function using the nanotube diameter of $1.60 \mathrm{~nm}$.

The helicity of the carbon nanotube can also be deduced accurately from its electron diffraction pattern by either measuring the relative twist angle ${ }^{13}$ or the ratio of the layer line spacings. ${ }^{16}$ Given the experimental limitations, using the ratio of layer line spacings would give rise to results of the highest accuracy. We measured $2 D_{1}$, the distance between the top and the bottom layer lines, $2 D_{2}$, the distance between the two middle layer lines, and $2 D_{3}$, the distance between the layer lines next to the equatorial layer line, respectively, as indicated in Fig. 2(b). The helicity $\alpha$ of this nanotube is calculated from the following equation: ${ }^{16}$

$$
\alpha=\tan ^{-1}\left(\left(2 D_{2}-D_{1}\right) / \sqrt{3} D_{1}\right) .
$$

The major error in the measurement of helicity comes from the errors in the measurement of the layer line spacings $D_{1}$ and $D_{2}$. In the present measurement, $\sigma_{1}$ and $\sigma_{2}$, the errors of measuring $D_{1}$ and $D_{2}$, respectively, are all $0.009 \mathrm{~nm}^{-1}$. The error in the deduction of the helicity is therefore

$$
\sigma_{\alpha}=\frac{2 \cos ^{2} \alpha}{\sqrt{3} D_{1}} \sqrt{\left(D_{2} / D_{1}\right)^{2} \sigma_{1}^{2}+\sigma_{2}^{2}},
$$

which would lead the maximum of error in the deduction of helicity to be $0.08^{\circ}$. The helicity of the nanotube shown in Fig. 2(a) is therefore $24.50^{\circ} \pm 0.08^{\circ}$, whose crystallographic indices are $(14,10)$. It is a semiconducting nanotube.

\section{RESULTS AND DISCUSSION}

Figure 3(a) shows a map of all carbon nanotubes of diameter from 0.5 to $3.0 \mathrm{~nm}$, in which the metallic tubules are represented by solid circles and the semiconducting ones by triangles. There are 458 carbon nanotubes of different indices $(u, v)$ within this range. The shaded area covers all nanotubes of diameter from 1.20 to $1.65 \mathrm{~nm}$, within which the abovediscussed NBED technique can assign the $(u, v)$ indices unambiguously with the accuracies of $\pm 1 \%$ in diameter measurement and $\pm 0.08^{\circ}$ in helicity measurement. There are total 70 nanotube species falling within this range.

To avoid selective sampling, we chose randomly isolated SWNTs for acquiring NBED data. In experiment we have characterized a total of 124 individual carbon nanotubes with 

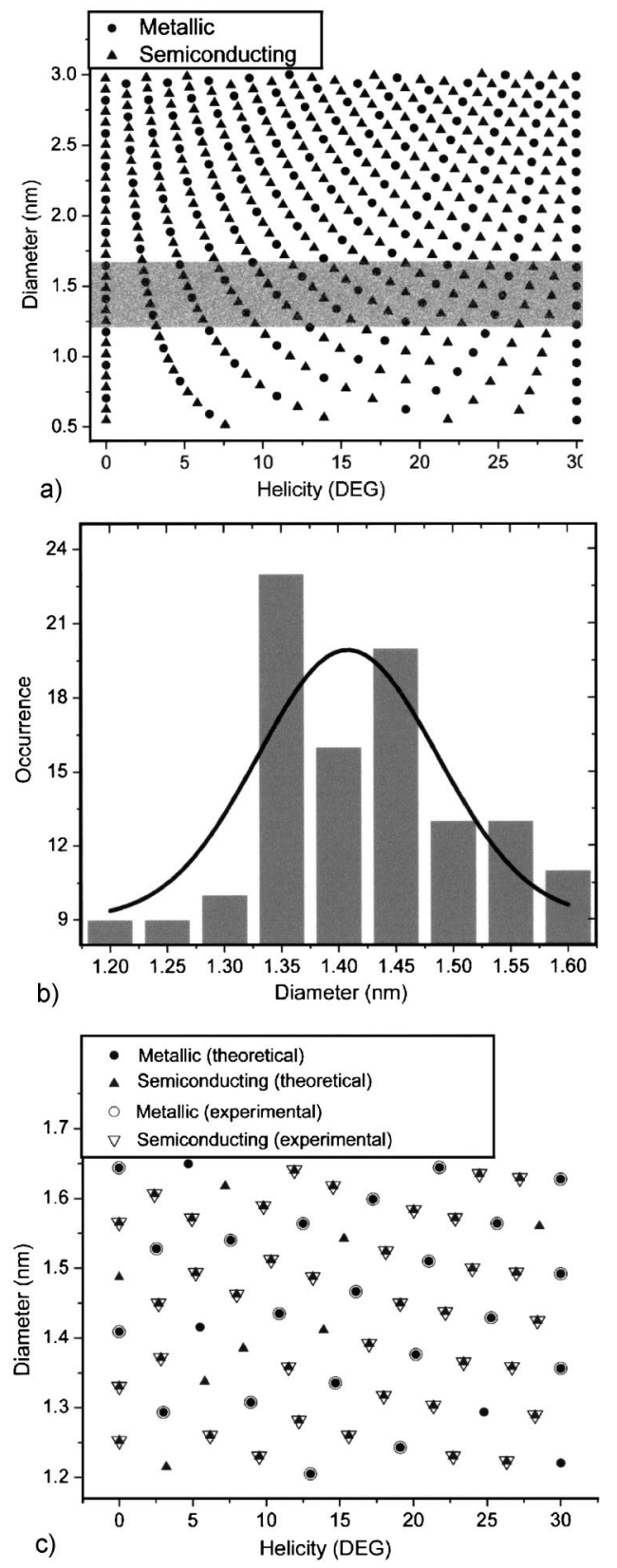

FIG. 3. (a) All 458 nanotube species with diameter from 0.8 to $3.0 \mathrm{~nm}$. Solid circles and triangles represent metallic and seminconducting nanotubes, respectively. (b) Diameter distribution of the 124 individual SWNTs examined experimentally. A Gaussian fit of the diameter distribution was represented by the black curve, in which the peak is around $1.38 \mathrm{~nm}$ with a standard deviation of 0.1 $\mathrm{nm}$. (c) Portion of the shaded region in (a), which has been characterized experimentally. 58 out of the 70 nanotube species were observed, among which 21 are metallic and 37 are semiconducting.

diameters in the range from 1.20 to $1.65 \mathrm{~nm}$, as shown in Fig. 3(b). The average diameter of the observed carbon nanotubes is $1.38 \mathrm{~nm}$, the same as the SWNTs produced by laser ablation. $^{25}$

Among the total 70 different nanotubes in this range, depicted in Fig. 3(c), the solid circles and triangles represent the metallic and semiconducting nanotubes, respectively. There are 25 metallic and 45 semiconducting nanotube spe-
TABLE I. List of the $(u, v)$ indices of the 124 examined nanotubes. Column $\mathrm{O}$ indicates the number of encounters of the corresponding nanotube species.

\begin{tabular}{llllll}
\hline \hline$(u, v)$ & $\mathrm{O}$ & $(u, v)$ & $\mathrm{O}$ & $(u, v)$ & $\mathrm{O}$ \\
\hline$(18,0)$ & 1 & $(16,0)$ & 2 & $(13,5)$ & 2 \\
$(21,0)$ & 1 & $(17,0)$ & 2 & $(14,6)$ & 4 \\
$(19,1)$ & 2 & $(19,0)$ & 0 & $(13,6)$ & 2 \\
$(16,1)$ & 1 & $(20,0)$ & 1 & $(15,7)$ & 3 \\
$(20,2)$ & 0 & $(20,1)$ & 1 & $(14,7)$ & 3 \\
$(17,2)$ & 0 & $(18,1)$ & 2 & $(15,8)$ & 2 \\
$(18,3)$ & 3 & $(17,1)$ & 4 & $(12,7)$ & 3 \\
$(15,3)$ & 1 & $(15,1)$ & 0 & $(13,8)$ & 5 \\
$(16,4)$ & 3 & $(19,2)$ & 2 & $(11,7)$ & 2 \\
$(17,5)$ & 2 & $(18,2)$ & 5 & $(14,9)$ & 3 \\
$(13,4)$ & 3 & $(16,2)$ & 0 & $(12,8)$ & 2 \\
$(14,5)$ & 2 & $(15,2)$ & 1 & $(13,9)$ & 1 \\
$(15,6)$ & 5 & $(19,3)$ & 0 & $(14,10)$ & 2 \\
$(16,7)$ & 1 & $(17,3)$ & 1 & $(10,8)$ & 1 \\
$(12,6)$ & 1 & $(16,3)$ & 0 & $(11,9)$ & 4 \\
$(13,7)$ & 3 & $(14,3)$ & 2 & $(12,10)$ & 1 \\
$(14,8)$ & 3 & $(18,4)$ & 1 & $(13,11)$ & 2 \\
$(15,9)$ & 1 & $(17,4)$ & 1 & $(10,9)$ & 2 \\
$(11,8)$ & 0 & $(15,4)$ & 2 & $(11,10)$ & 3 \\
$(12,9)$ & 4 & $(18,5)$ & 1 & $(12,11)$ & 0 \\
$(13,10)$ & 2 & $(14,4)$ & 1 & $(16,5)$ & 2 \\
$(9,9)$ & 0 & $(11,11)$ & 1 & $(15,5)$ & 0 \\
$(10,10)$ & 4 & $(12,12)$ & 1 & $(17,6)$ & 1 \\
$(16,6)$ & 0 & & & & \\
\hline \hline
\end{tabular}

cies in this diameter range. It should be noted that the nanotubes are not evenly distributed, for instance, along the zigzag nanotube line (helicity is $0^{\circ}$ ), there are more nanotubes than at other helicities. Metallic nanotubes, with $n-m$ evenly divisible by 3 , intertwine with semiconducting nanotubes in the map.

For the 124 isolated SWNTs examined randomly (a complete list is given in Table I), 58 nanotubes of different structure have been identified, covering more than $80 \%$ of all 70 configurations. The metallic and semiconducting nanotubes have a number ratio of $21 / 37$, which is in accordance with the population ratio of $25 / 45$. It shows that there is no preference of either metallic nanotubes or semiconducting nanotubes. However, some of the individuals were observed more often than others, for example, carbon nanotube $(13,8)$, a semiconducting nanotube, was encountered five times.

Among the 70 possible nanotubes in the diameter range between 1.20 and $1.65 \mathrm{~nm}$, the experimental observations showed that there was no strong preference in the distribution of helicity, while noticeable fluctuations in the occurrence of some helicities were visible as shown in Fig. 4(a). For example, the observed zigzag nanotubes are equal to that of armchair nanotubes. Ab initio calculations of the total energy of both chiral and achiral nanotubes indicate that the energy difference is smaller than $20 \mathrm{meV} /$ atom between 

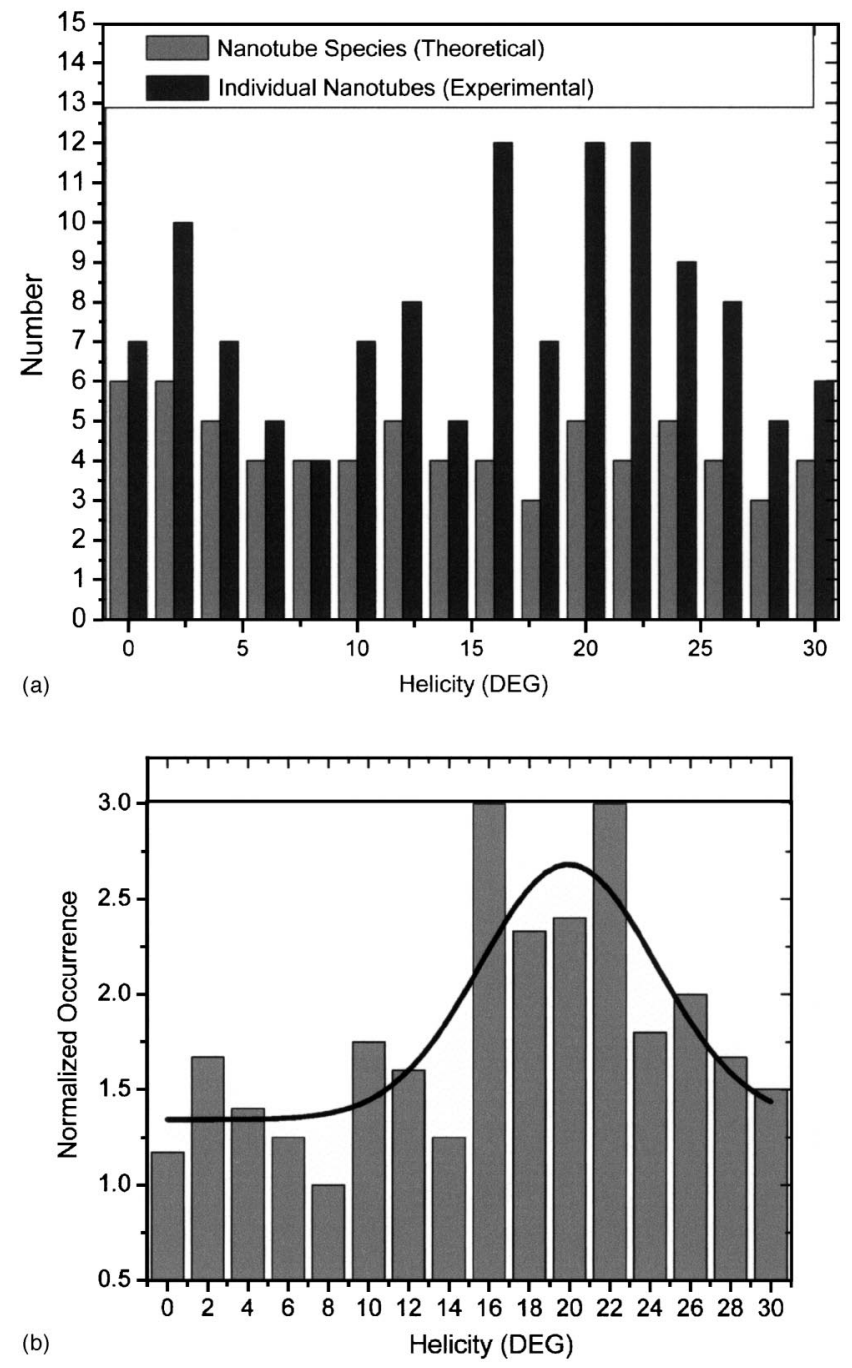

FIG. 4. Helicity distribution of the 124 individual SWNTs characterized experimentally. (a) Histogram showing the number of nanotube species (gray column) and the number of the observed individual nanotubes (black column) with different helicities, plotted in intervals of $2^{\circ}$. (b) Histogram showing normalized number (normalized by dividing the observed number of nanotubes by the number of nanotube species at each helicity) of characterized individual nanotubes with different helicities. Nanotubes of helicities $15^{\circ}-30^{\circ}$ were slightly favored, attributed to the higher growth rate of these nanotubes.

nanotubes of different structures. This suggests that, energetically, all carbon nanotubes could be formed without strong preferences. Basing on the equal petition of energy in the formation of nuclei, we suggest that the distribution of helicity among the chiral nanotubes be uniform and occur randomly as discussed in the polyyne ring model. ${ }^{26}$ Once the nuclei of carbon nanotubes were formed, carbon atoms or other species such as dimers and trimers were added to the open ends with the assistance of metal catalysts. Continuous addition of the various carbon species promotes the growth of carbon nanotubes.

However, detailed examination of the helicity distribution reveals that there was a slightly favored distribution of helicities in the experimental measurement. This observation is

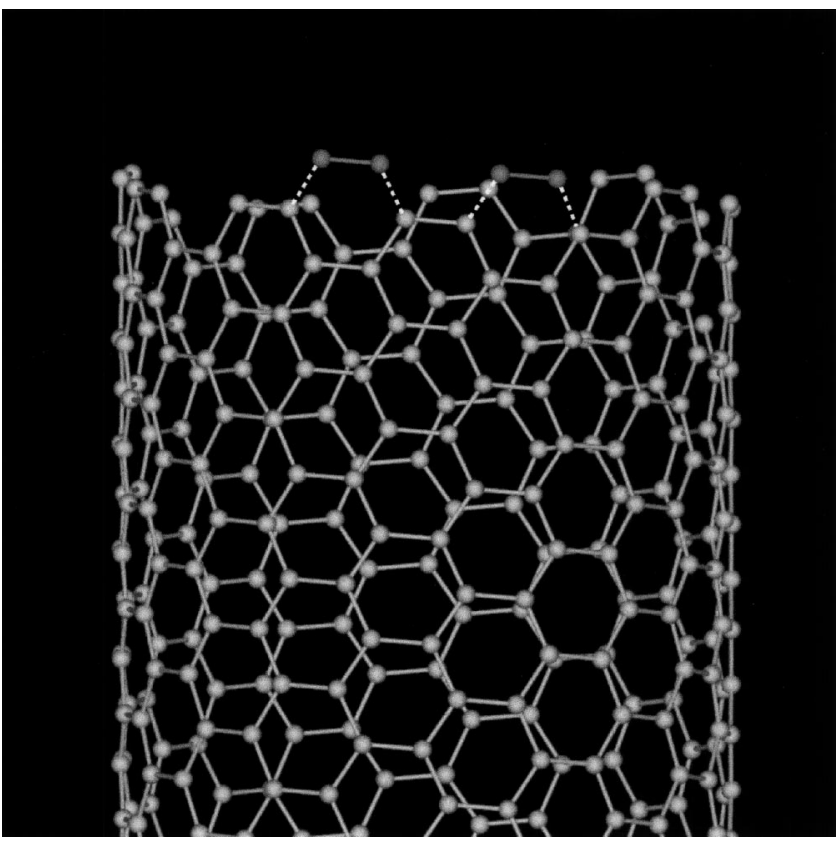

FIG. 5. Schematic illustrating the faster growth rate of nanotubes of helicities $15^{\circ}-30^{\circ}$, where additions of newly arrived dimers (dark-gray colored) would facilitate higher growth rate. The dotted lines denote new bonds.

better reflected in the plot of the normalized distribution of observed nanotubes with respect to helicity, as shown in Fig. 4(b). A Gaussian peak, also shown in the figure, was observed around the helicity of $20^{\circ}$ with a standard deviation of $4^{\circ}$. Actually, for the examined sample, more than $40 \%$ of the nanotubes observed are located between the helicities of $16^{\circ}$ and $24^{\circ}$. We suggest that the favored helicity distribution of nanotubes be attributed to the different growth rates of nanotubes of different structures, though the nucleation rates of various nanotube species had a uniform distribution. The original nuclei of carbon nanotubes may be either chiral or achiral. However, the higher concentration of carbon dimers available in the carbon plasma plume makes the growth of carbon nanotubes of helicity near the armchair structure faster than other configurations as schematically illustrated in Fig. 5, where the two newly added dimers were forming covalent bonds (indicated by dotted lines) with the alreadygrown structure. It is this kinetics factor that resulted in the uneven distribution of observed configuration of carbon nanotubes. We believe that control and manipulation of the distribution of helicity of carbon nanotubes can be exercised by tuning the concentrations of the various carbon species (single atoms, dimers, trimers, etc.) in the carbon source from which the growth of carbon nanotubes is accomplished.

\section{CONCLUSIONS}

Kinematical theory of electron diffraction has been developed and applied to obtain the chiral indices of single-walled carbon nanotubes. Although the diameter of a SWNT can be measured directly from the HRTEM image, the intensity dis- 
tribution on the equatorial layer line offers higher accuracy in the measurement. Furthermore, the chiral indices of a SWNT can be derived accurately from the intensity distribution on the principal layer lines. In total the diameter and helicity of 124 randomly isolated SWNTs belonging to 58 nanotube species have been determined unambiguously. The determined nanotube structures are mapped with respect to their diameters and helicities and compared with the theoretical mapping of 70 possible carbon nanotube species in the range of 1.20 to $1.65 \mathrm{~nm}$ in diameter with all possible helici- ties. By examining the histogram of helicities, we find that, while certain nanotubes were observed slightly more often than others, the overall feature showed a rather uniform distribution in occurrence. Basing on a nucleation-and-growth model, we suggest that the uniform distribution of helicity be originated from the weak dependence on helicity of the formation energy of carbon nanotubes, while the growth prefers slightly the structure with helicity $15^{\circ}-30^{\circ}$ for which the addition of carbon dimers would facilitate the growth of carbon nanotubes.
*Corresponding author. Email address. lcqin@physics.unc.edu

${ }^{1}$ N. Hamada, S. Sawada, and A. Oshiyama, Phys. Rev. Lett. 68, 1579 (1992).

${ }^{2}$ R. Saito, M. Fujita, G. Dresselhaus, and M. S. Dresselhaus, Appl. Phys. Lett. 60, 2204 (1992).

${ }^{3}$ L.-C. Qin, X. Zhao, K. Hirahara, Y. Miyamoto, Y. Ando, and S. Iijima, Nature (London) 408, 50 (2000).

${ }^{4}$ A. M. Rao, E. Richter, S. Bandow, B. Chase, P. C. Eklund, K. A. Williams, S. Fang, K. R. Subbaswamy, M. Menon, A. Thess, R. E. Smalley, G. Dresselhaus, and M. S. Dresselhaus, Science 275, 187 (1997).

${ }^{5}$ A. Jorio, M. A. Pimenta, A. G. Souza Filho, R. Saito, G. Dresselhaus, and M. S. Dresselhaus, New J. Phys. 5, 139.1 (2003).

${ }^{6}$ S. M. Bachilo, M. S. Strano, C. Kittrell, R. H. Hauge, R. E. Smalley, and R. B. Weisman, Science 298, 2361 (2002).

${ }^{7}$ M. S. Strano, S. K. Doorn, E. H. Haroz, C. Kittrell, R. H. Hauge, and R. E. Smalley, Nano Lett. 3, 1091 (2003).

${ }^{8}$ J. W. G. Wildoer, L. C. Venema, A. G. Rinzler, R. E. Smalley, and C. Dekker, Nature (London) 391, 59 (1998).

${ }^{9}$ T. W. Odom, J.-L. Huang, P. Kim, and C. M. Lieber, Nature (London) 391, 62 (1998).

${ }^{10}$ L.-C. Qin, T. Ichihashi, and S. Iijima, Ultramicroscopy 67, 181 (1997).

${ }^{11}$ L.-C. Qin, S. Iijima, H. Kataura, Y. Maniwa, S. Suzuki, and Y. Achiba, Chem. Phys. Lett. 268, 101 (1997).

${ }^{12}$ J. M. Cowley, P. Nikolaev, A. Thess, and R. E. Smalley, Chem.
Phys. Lett. 265, 379 (1997).

${ }^{13}$ L.-C. Qin, Chem. Phys. Lett. 297, 23 (1998).

${ }^{14}$ J.-F. Colomer, L. Henrard, Ph. Lambin, and G. Van Tendeloo, Eur. Phys. J. B 27, 111 (2002).

${ }^{15}$ M. Kociak, K. Suenaga, K. Hirahara, Y. Saito, T. Nakahira, and S. Iijima, Phys. Rev. Lett. 89, 155501 (2002).

${ }^{16}$ M. Gao, J. M. Zuo, R. D. Twesten, I. Petrov, L. A. Nagahara, and R. Zhang, Appl. Phys. Lett. 82, 2703 (2003).

${ }^{17}$ J. M. Zuo, I. Vartanyants, M. Gao, R. Zhang, and L. A. Nagahara, Science 300, 1419 (2003).

${ }^{18}$ C. Journet, W. K. Maser, P. Bernier, A. Loiseau, M. Lamy de la Chappelle, S. Lefrant, P. Deniard, R. Lee, and J. E. Fischer, Nature (London) 388, 756 (1997).

${ }^{19}$ L. A. Girifalco, M. Hodak, and R. S. Lee, Phys. Rev. B 62, 13104 (2000).

${ }^{20}$ L.-C. Qin, J. Mater. Res. 9, 2450 (1994).

${ }^{21}$ A. A. Lucas, V. Bruyninckx, and Ph. Lambin, Europhys. Lett. 35, 355 (1996).

${ }^{22}$ Z. Liu and L.-C. Qin, Chem. Phys. Lett. 400, 435 (2004).

${ }^{23}$ Z. Liu and L.-C. Qin, Chem. Phys. Lett. 402, 202 (2005).

${ }^{24}$ B. W. Smith and D. E. Luzzi, J. Appl. Phys. 90, 3509 (2001).

${ }^{25}$ A. Thess, R. Lee, P. Nikolaev, H. Dai, P. Petit, J. Robert, C. Xu, Y. H. Lee, S. G. Kim, D. T. Colbert, G. Scuseria, D. Tomanek, J. E. Fischer, and R. E. Smalley, Science 273, 483 (1996).

${ }^{26}$ C.-H. Kiang and W. A. Goddard III, Phys. Rev. Lett. 76, 2515 (1996). 